\section{Saures Nitrit setzt Genitalwarzen zu}

\begin{abstract}
Die Kombination von Nitrit und Zitronensäure eignet sich offenbar zur Therapie gegen Genitalwarzen, so die Ergebnisse einer Studie mit knapp 300 Patienten. Die Teilnehmer wurden in vier Gruppen über drei Monate hinweg behandelt. Die erste Gruppe trug zwei Placebocremes auf. Damit wurden 14\% ihre Warzen los. In der zweiten Gruppe bekamen die Patienten eine niedrigdosierte Nitritcreme (3\%) und eine niedrigdosierteZitronensäurecreme $(4,5 \%)$. $15 \%$ der Patienten waren nach zwölf Wochen warzenfrei. Mit 6\% Nitrit und 9\% Zitronensäure gelang dies $23 \%$, und mit $9 \%$ Nitrit und 9\% Zitronensäure verschwanden die Warzen bei 31\% der Patienten.
\end{abstract}

Ormerod A D et al. Jama Dermatol 2015; 151(8):854-861

\section{Alopecia areata durch Zinkmangel?}

Bei Patienten mit Alopecia areata sind die Zinkspiegel im Serum signifikant verringert. Zudem korrelieren sie invers mit der Therapieresistenz. Und: Je niedriger die Serumspiegel sind, umso ausgeprägter ist die Alopezie, wie die Ergebnisse einer Studie mit insgesamt 100 Teilnahmern bestätigen. Als pathogenetischen Zusammenhang vermuten Wissenschaftler unter anderem eine Einschränkung der T-Zellfunktion. Darüber hinaus verlangsamt die Unterversorgung der Zellen mit Zink das Wachstum der Haare, die außerdem leichter abbrechen. Die Patienten könnten möglicherweise von einer Supplementierung profitieren.

Abdel Fattah NS et al. Int J Dermatol 2015 (online first)

\section{Wunden schneller heilen lassen}

Schlecht heilende Wunden sind für Patienten nicht nur mit Schmerzen, eingeschränkter Mobilität und Infektionsgefahr verbunden, sondern häufig auch mit frustranen Langzeittherapien und sozialer Isolation. Um dies zu vermeiden und maximalen Heilungserfolg zu erzielen, hat sich die interdisziplinäre und interaktive Wundtherapie z.B. mit Radiologen und Gefäßchirurgen bewährt. Gehen Sie ins Detail und lernen Sie mehrim Schwerpunkt "Dermatologie"!

Claudia Daniels Redakteurin

\title{
Psoriasispatienten in die Augen schauen!
}

Bei Patienten mit Psoriasis sollte man offenbar auch die Augen im Blick behalten. Nicht selten findet sich bei ihnen nämlich eine Uveitis. Die Assoziation zwischen beiden Erkrankungen hat eine aktuelle dänische Kohortenstudie an einer großen Patientenzahl bestätigt. In dieser Studie war das Risiko für Patienten mit Psoriasis und Psoriasisarthritis, eine Entzündung der mittleren Augenhaut $\mathrm{zu}$ entwickeln, generell erhöht. Besonders gefährdet scheinen demnach Patienten mit einer Spondylitis zu sein, sie hatten ein mehr als achtfach erhöhtes
Risiko. Die Psoriasisarthritis war mit einem 2,5-fach erhöhten, die milde sowie schwere Psoriasis mit einem um $40 \%$ erhöhten Uveitisrisiko assoziiert. Auch umgekehrt ließ sich ein Zusammenhang feststellen. So war es für Uveitispatienten wahrscheinlicher, an einer milden oder schweren Psoriasis oder einer Psoriasisarthritis zu erkranken, als für Menschen ohne diese Augenerkrankung. Die gesamte Studienpopulation umfasste 5.508.878 dänische Bürger.

Egeberg A et al. JAMA Dermatol. 2015 (online first)

\section{Malignes Melanom: Neuer Standard etabliert sich}

Bei malignen BRAF-mutierten Melanomen wird sich wohl die Kombination aus einem BRAF-Inhibitor und einem MEKInhibitor als Standardtherapie etablieren. Zumindest sprechen dafür die neuesten Ergebnisse der COMBI d-Studie, denn die Kombination aus einem BRAF- und MEK-Inhibitor konnte bei der Behandlung von Patienten mit BRAF-mutierten malignen Melanomen erneut überzeugen. Letztlich verlängerte die Kombinationstherapie das progressionsfreie Über- leben im Vergleich zur Monotherapie sogar deutlicher, als es in der ersten Analyse mit 9,3 Monaten zu sehen war: nämlich um elf Monate im Vergleich zu 8,8 Monaten unter dem BRAF-Inhibitor. Das Sterberisiko für Patienten, die beide Kinasehemmer eingenommen hatten, reduzierte sich signifikant um 29\%. Die Überlebensrate betrug nach einem Jahr 74\% und nach zwei Jahren 51\%.

Long GV et al. The Lancet 2015; 386:444-451

\section{Der Hype um Sonnenbrand-Tattoos}

Eine Frau hat ein Herzchen auf der Haut, ein junger Mann zeigt sich mit „Batman“-Symbol auf der Brust. Für die Ewigkeit sind diese „Tattoos“, die sie im Internet präsentieren, aber mitnichten. Denn die Bildchen sind nicht vom Tätowierer gestochen - sondern von der Sonne gebrannt. Unter dem Hashtag \#sunburnart (Sonnenbrand-Kunst) veröffentlichen zurzeit vor allem junge Leute solche Fotos von sich im Netz. Anbieter wie „Funtantattoo“ verkaufen entsprechende Schablonen bereits über das Internet in dutzende Länder. Hautärzte warnen jedoch vor dem Phänomen. „Das Problem ist, dass es im Rahmen von solchen kräftigen Sonnenbränden auch zu Mutationen an genetischen Sequenzen kommt, die für die Unterdrückung von Tumor-Reaktionen verantwortlich sind“, so Dr. Thomas Dirschka, Dermatologe aus Wuppertal. Deswegen erhöhe jeder Sonnenbrand das Risiko für Hautkrebs, warnt er. Auch ein Herzchen auf dem Bauch sei davon nicht ausgenommen.

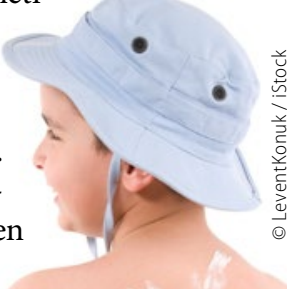

